\title{
Requirements for the design and implementation of checklists for surgical processes
}

\author{
E. G. G. Verdaasdonk $\cdot$ L. P. S. Stassen · \\ P. P. Widhiasmara $\cdot$ J. Dankelman
}

Received: 4 November 2007/ Accepted: 30 May 2008/Published online: 18 July 2008

(C) The Author(s) 2008

\begin{abstract}
Background The use of checklists is a promising strategy for improving patient safety in all types of surgical processes inside and outside the operating room. This article aims to provide requirements and implementation of checklists for surgical processes.

Methods The literature on checklist use in the operating room was reviewed based on research using Medline, Pubmed, and Google Scholar. Although all the studies showed positive effects and important benefits such as improved team cohesion, improved awareness of safety issues, and reduction of errors, their number still is limited. The motivation of team members is considered essential for compliance. Currently, no general guidelines exist for checklist design in the surgical field. Based on the authors' experiences and on guidelines used in the aviation industry, requirements for the checklist design are proposed. The design depends on the checklist purpose, philosophy, and method chosen. The methods consist of the "call-doresponse" approach," the "do-verify" approach, or a combination of both. The advantages and disadvantages of paper versus electronic solutions are discussed. Furthermore, a step-by-step strategy of how to implement a checklist in the clinical situation is suggested.
\end{abstract}

E. G. G. Verdaasdonk ( $₫)$ - L. P. S. Stassen •

P. P. Widhiasmara · J. Dankelman

Department of BioMechanical Engineering,

Faculty of Mechanical, Maritime, and Materials Engineering,

Man Machine Systems Group, Delft University of Technology,

Mekelweg 2, 2628, CD, Delft, The Netherlands

e-mail: e.g.g.verdaasdonk@tudelft.nl

E. G. G. Verdaasdonk · L. P. S. Stassen

Department of Surgery, Reinier de Graaf Gasthuis, Delft,

The Netherlands
Conclusions The use of structured checklists in surgical processes is most likely to be effective because it standardizes human performance and ensures that procedures are followed correctly instead of relying on human memory alone. Several studies present promising and positive first results, providing a solid basis for further investigation. Future research should focus on the effect of various checklist designs and strategies to ensure maximal compliance.

Keywords Endoscopy - Human error - Safety checklist · Instruments $\cdot$ Technical $\cdot$ Human/robotic

The increased complexity of the operating room forces medical professionals to put more effort into improving surgical safety. The report "To Err Is Human: Building a Safer Health System" emphasizes the occurrence of errors in medicine [1]. This report estimates that at least 44,000 people die annually because of medical errors in the United States, but this number may be as high as 98,000 . A recent study conducted in the Netherlands showed that more than 1,700 patients die due to medical errors each year [2]. Leape [3] showed that a common site for adverse events in the hospital is the operating room. In addition, most of these adverse events were considered preventable.

Several reports have underscored the importance of Reason's [4] "system approach," which takes measures to reduce adverse events in the hospital, instead of the persons' approach [5-8]. According to Reason's theory, safety in complex environments (e.g., the operating room) relies on multiple system defenses such as the organizational structure, protocols, training of professionals, and quality of equipment or technology. When the defenses fail or are 
flawed, an accident is bound to happen. With the system approach, the conditions under which individuals work are thoroughly investigated, and efforts are made to build strong defenses to avoid human errors or diminish their effects.

In a study by Undre et al. [9] investigating 50 surgical procedures, significant steps were being missed, which at the very least eroded safety margins. Frequent failure to check both surgical and anesthetic equipment occurred as well as failure to confirm the procedure verbally. In twothirds of the cases, delays or changes occurred, and in oneeighth of the procedures, the patient notes were missing.

Recently, problems related to the technical equipment during minimally invasive surgery (MIS) were studied [10]. This research showed that although no adverse events occurred, the incidence of problems with equipment and instruments was strikingly high during routine surgical procedures. Each time an incident occurred, the operation flow was obstructed and valuable time was lost. The majority of these problems could have been prevented by correct use and preparation of the equipment before the actual procedure. Besides training of personnel, incorporation of a short checklist before the start of each surgical procedure was recommended.

Parallel to the aviation industry, checklist use may be a promising strategy in health care. A checklist could serve as a structural memory aid, helping surgical crews to check and confirm the readiness of the equipment before the operation begins. However, physical appearance and user interaction should be carefully designed so that the checklist serves its purpose. For example, a checklist that is too long and difficult to read or one that uses ambiguous terminology may have a negative effect on the task performance instead of improving it. On the other hand, if the checklist is too short and does not incorporate all critical steps, it may have no effect at all. Furthermore, a clear strategy to incorporate a checklist in the clinical situation is needed.

This report aims to provide general requirements for the design and implementation of checklists for surgical processes.

\section{Methods}

The existing literature on checklist use in the operating room was studied. The Google Scholar, Medline, and Pubmed databases were searched using the search terms "checklist," "operating room," "surgery," and "safety." Books or publications in peer-reviewed journals between January 1980 and June 2007 were included. Only publications in the English language were considered.

Publications included clearly addressed use of the checklist to improve the quality of care, team communication, patient safety, or use of equipment and instruments in the operating room or the effect of a structured checklist in the operating room.

After the results of the literature review, the general and the physical requirements for the checklist design are presented. Requirements for the checklist design are based on our own experiences and on guidelines used in the aviation industry. The advantages and disadvantages of paper and electronic checklists are brought to attention. A flow chart, following a number of practical steps, was developed for incorporation into the checklist. Finally, we discuss the broader context of the checklist for surgical processes and future research.

\section{Results}

The term "checklist" produced 7,429 hits in Pubmed and Medline and 53,200 hits in Google Scholar. The search was narrowed down by adding the term "operating room," which produced 27 publications in Pubmed and Medline. In Google Scholar, "surgery," "safety," and "protocol" also were added. The search was further narrowed by including only publications and citations from medicine, pharmacology, and veterinary science, which resulted in 271 hits.

All literature references were manually checked for relevance. Double references were excluded. A total of eight publications were considered relevant to checklist use in the operating room. Cross-linking of the references identified eight additional publications and two guidelines from an electronic source. No randomized controlled trials were found.

\section{Checklist use in the operating room}

The use of a checklist as an evaluation or audit tool in the operating room is not an entirely new concept. In July 2004, the Joint Commission on Accreditation of Healthcare Organizations (JCAHO) mandated the Universal Protocol for the prevention of wrong-site, wrong-side, wrongprocedure, and wrong-person surgery for all Joint Commission-accredited organizations [11]. The protocol consists of guidelines for a preoperative verification process, marking of the operative site, and a "time out" immediately before start of the procedure [12]. During the time out, critical information about the patient and the surgical procedure planned is checked by the surgical team members.

The goals and the content of the JCAHO protocol are stated explicitly. The use of a structured checklist also is recommended. However, criteria for the format of the protocols or checklists are not given. A critical report, conducted before the Universal Protocol was mandated, 
stated that wrong-site surgery is exceedingly rare (1 in 112,994 operations) and that hospital protocol design varied significantly [13]. Nevertheless, under optimal conditions, the JCAHO protocol could have prevented twothirds of the examined cases.

Currently, limited evidence exists to prove that interventions such as the Universal Protocol are effective [14]. Furthermore, hospitals are facing difficulties evaluating the effect of their policies and whether they are preventing adverse events.

Lingard [15] developed a checklist to enhance performance in the operating room and investigated the feasibility of a preoperative checklist as an aid to communication between surgical team members. The list was designed by a research team of experts from various backgrounds including a communication researcher, a cognitive psychologist, nurses, an anesthesiologist, a surgical trainee, and research staff. This study prospectively included 18 surgical procedures. Before each procedure, the surgical team was asked to conduct a discussion according to the checklist.

The data collected through observation and interviews showed that the checklist was feasible, providing positive effects on information exchange and addressing educational issues and team cohesion. The surgeons' commitment was particularly important to successful checklist implementation. All the participants felt that completion of the checklist before setup of the procedure was optimal. Further research was suggested to determine the sustainability and generalization of checklist intervention and to investigate its impact on patient safety.

With their Safety Attitudes Questionnaire (SAQ), Makary et al. [16] evaluated the impact of operating briefings on coordination of care and risk for wrong-site surgery. The questionnaire was administered before and after initiation of an operating room briefing program with a previously developed structured checklist [17]. The results showed that the personnel (surgeons, anesthesiologists, nurses) subjectively perceived a significantly reduced risk for wrong-site surgery and improved collaboration. The briefings improved awareness of the surgical site and side targeted for surgery. Furthermore, during implementation of the program, the quality of the briefings improved. Although the authors acknowledge that their study does not provide evidence that the rate of wrong-site surgery decreased, they point out that the SAQ scores may well be associated with clinical improvements and a better outcome in the operating room. Furthermore, they emphasize the role of a "champion physician" to facilitate development and encourage the briefing protocol.

Leonard et al. [18] reported that surgical teams who implemented a perioperative team briefing process at a nonprofit hospital in the United States achieved positive results. More detailed results were presented by DeFontes [19]. The briefing chart was broken down into four sections: surgeon, circulating nurse, scrub nurse, and anesthesiologist. Each section member had to elucidate several items in a given case before the surgery. In contrast to Lingard's study, the briefing was performed at the moment the patient was anesthetized because the team members had decided that this was the only time they all were consistently present.

Since the introduction of the briefing process, wrong-site surgeries have decreased from 3 to 0 , nursing turnover has dropped by $16 \%$, and employee satisfaction (measured with the Safety Attitudes Questionnaire) has increased by $19 \%$ [19]. Perceptions of the safety climate in the operating room have increased from "good" to "outstanding" [19].

Anesthesia is an operating room domain that has been using a checklist as a safety aid for some time. Various checklists have been developed, especially for checking the anesthetic machinery. Studies show that various concepts detect most faults effectively [20-23]. A simulator also exists to train detection of equipment failure [24].

One study showed that an electronic checklist is superior to the standard Food and Drug Administration (FDA)approved paper checklist list in detecting equipment faults [25]. However, all studies evaluating equipment checklists for anesthetic machinery acknowledge that all faults could not always be detected. Hence, no checklist guarantees $100 \%$ accuracy.

Kendell and Barthram [26] observed the implementation of a safety checklist for anesthesia equipment based on revised guidelines. The observation, conducted in a district general hospital for 6 weeks, resulted in the completion of 132 checklists. Analysis showed that at least $82.5 \%$ of the completed checklists pointed out a fault in the anesthesia equipment. The results underscored the ability of a safety checklist to detect system failures. A constraining factor mentioned by Kendell and Barthram [26] is time required to complete the checklist. Time governs willingness and compliance in the use of checklists. Time-consuming checklists can result in failure of checklist completion. Hence, an important consideration in checklist design is its length and practicability.

Another study conducted by Hart and Owen [27] in the anesthesia field demonstrated the function of a checklist as a memory aid tool. This study investigated the possibility of using a verbal checklist to assist the anesthesiologist administering general anesthesia during cesarean delivery. An electronic checklist (voice controlled) and a highfidelity anesthesia simulator together with a predetermined scenario were used to collect data. The subject also filled out a questionnaire.

Most of the subjects thought the checklist was useful, although only a minority wished to use it in practice. 
Completion time for the checklist also was mentioned as an important factor to be considered for further implementation. Remarkably, $60 \%$ of the participants preferred a written checklist to the verbal checklist. The problem with electronic checklists lies with the technology used to build the interface device. In the study by Hart and Owen [27], several subjects had difficulty understanding the voice synthesized by the device, so the written checklist was preferred to the verbal checklist.

The use of a checklist for laparoscopic equipment has been mentioned previously. Meijer [28] pointed out the potential benefits of a checklist for the laparoscopic equipment and described some critical checkpoints. It also was suggested that to ensure the proper state and good quality of laparoscopic equipment, a preoperative checklist should become a standard. However, explicit design requirements were not described.

In summary, the number of studies on checklist use in the operating room is limited. Several studies present promising and positive first results on improvement of team coherence and reduction of errors, providing a solid basis for further investigation. Although guidelines for the critical content of some checklists are available, no general guidelines for the development of checklists are provided. Moreover, the requirements for actual checklist design have not been investigated previously.

\section{Checklist requirements}

\section{General purpose of checklists}

Checklists are used commonly in the aviation industry. Requirements and guidelines for checklist design are provided by the Federal Aviation Authorities (FAA) and the Civil Aviation Authority (CAA) on the Internet [29, 30]. These guidelines provide detailed information about the specifications for the checklists such as the checklist layout, format, letter fonts, and physical construction of the document.

In health care, the most important function of a checklist is to ensure the correct execution of a given procedure or tasks. It forms the first step in standardization of procedural performance.

Based on the reviewed literature and reports from aviation [31, 33], a checklist in the operating room should serve as

- a defense strategy to prevent human errors

- a memory aid to enhance task performance

- standardization of the tasks to facilitate team coordination

- a means to create and maintain a safety culture in the operation room
- support quality control by hospital management, government, and inspectors.

\section{Normal, non-normal, and emergency checklists}

In aviation, checklists are divided into three categories: normal, non-normal (also referred to as abnormal), and emergency checklists. In the medical field, a similar differentiation also is important because it influences the requirements for the final checklist design. In general, the requirements for the normal or routine checklist are less strict than for the emergency checklist. Non-normal and emergency checklists must contain each sequential step of a procedure, whereas the normal checklist typically is a listing of action items to be performed and does not necessarily represent each procedural step in sequential order.

\section{Checklist philosophy}

It is important to decide what checklist philosophy will be followed in terms of checklist design and the content that will be included. There are two approaches: the system engineering approach and the human performance approach.

With system engineering approach, all items involved in performing the task or setting up the equipment correctly should be checked. For example, in laparoscopic surgery, all instruments on the operating room table and all steps to setting up the equipment should be checked. Consequently, this results in a long checklist. From the human performance perspective, a detailed checklist is no guarantee of absolute safety because it carries the risk that the users will fail to use it correctly or choose not to use the list at all.

According to the human performance approach, only the critical items should be checked to overcome nuisance. An item is considered critical if failure to check it could lead to accidents. Nevertheless, deciding which items are critical is disputable because accident research in various high-risk environments has shown that small, seemingly unimportant incidents can have disastrous consequences [32].

It is important to consider human capabilities as well as human limitations in designing a checklist. For the medical field in general and surgical processes in particular, the best strategy may be to follow the human performance approach and start with the most essential checks.

\section{Checklist method}

Another important choice is the checklist method. Two dominant types can be distinguished: the "call-doresponse" (CDR) method, the "do-verify" (DV) method, or combinations of both [29, 30, 33]. 
The CDR checklist, also called the "do-list" or the "challenge-do-response checklist," uses a step-by-step "cookbook" approach [33]. In an aircraft, one crew member calls an item before the action is initiated. Then the action is taken, followed by verification that the action has been accomplished. This method is most effective when one crew member accomplishes the action and another verifies (cross-checks) that the action was taken. This cross-checking between team members keeps all personnel involved and has the advantage that all items are checked in a systematic manner. For the non-normal or emergency checklist, the CDR method is recommended, but it also has been used successfully for the normal checklist.

The CDR method can be translated to the operating room during critical steps of the procedure or identification of important "landmark" structures, for example, crosschecking the "critical view of safety" during laparoscopic cholecystectomy (identifying the cystic duct and artery going into the gallbladder) [34]. However, the disadvantage of this method is its rigidity, especially when the list is long. Team members cannot perform other tasks at the same time, and once the sequence has been interrupted, a skipped item can pass unnoticed [33]. The CDR method fits perfectly into the concept of what is referenced as "timeout procedure." Before the surgical procedure, the time out takes place in which critical items are checked between team members, for example, the name of the patient, the site of operation, and the potential allergies for disinfectants. These safety checks are already used in surgery, and several hospitals have already formalized them in their standard protocol.

With the DV method, also the called "cleanup" method, pilots configure an airplane according to memory in variable sequence. Then the checklist is read to verify that all items have been correctly accomplished. The advantage of this approach is that each individual team member can work independently, which enhances efficiency (a series of items is quickly performed) and balances the workload between team members. Most commonly used by commercial airlines [33], it is the method recommended for the normal checklist. The disadvantage of the DV method is the higher risk of items being missed than with the CDR method. In the operating room, the DV method can be useful for verifying all surgical instruments and for the setup of the laparoscopic equipment before the start of the procedure.

\section{Design requirements}

The checklist requirements recommended for surgical processes are summarized in Table 1. The recommendations are based on the guidelines from the CAA and FAA
[29, 30]. Furthermore, several other resources recommended by the aviation authorities $[31,33]$, combined with our own experience derived from a pilot study with a checklist, are used.

The most important requirements for the checklist design are consistency, clarity, and straightforwardness. A checklist should serve a clearly stated purpose and should be used intuitively. Some recommendations are very general and logical such as robustness and consistency. Others are more specific such as number of checks, binding, font size, font type, and spacing.

\section{Checklist solutions}

In the commercial aviation industry, many examples are available. This section focuses on the feasibility, advantages, and disadvantages of various checklist alternatives for surgical processes. Two major distinctions will be made between types of checklists: the paper and the electronic checklist.

\section{Paper checklist}

The first and probably the simplest solution is the paper checklist. The most important advantage of the paper checklist is its low technical complexity and high reliability. This form can be made into various sizes according to its purpose. Most paper-based checklists are portable and thus can be carried around. They are easily produced at relatively low cost and do not require additional infrastructure or technology for implementation. Furthermore, the paper checklist is highly reliable because it is independent of power supply, maintenance, or computer malfunction. This makes paper checklists very suitable for emergency and abnormal circumstances.

From an ergonomics point of view, a paper-based checklist usually is provided also with some kind of medium for ease of holding and writing such as a papersized cardboard with a clip on it.

It is important to be aware of the fact that paper checklist items in aviation are not marked when completed. These (cardboard or plasticized) checklists are used by the flight crew to perform important steps of their task. Moreover, checklist use seems to be second nature for pilots. Their entire training is aimed at correct execution of procedures, and checklist use is practiced extensively. Checks are called aloud, recorded by the voice recorder and, in case of an incident or accident, scrutinized for design faults or incorrect execution. This renders the marking of items in this field redundant. However, the disadvantage of reusable paper-based checklists without marking is that there is no memory of completed items. Another inherent disadvantage of the paper solution is its 
Table 1 Recommended requirements for the design of the surgical checklist

\begin{tabular}{|c|c|}
\hline Aspect & Requirement \\
\hline \multicolumn{2}{|l|}{ General } \\
\hline Consistency & $\begin{array}{l}\text { o Consistent format maintained for each checklist within hospitals and within surgical procedures } \\
\text { o Abbreviations consistent on all checklists }\end{array}$ \\
\hline Quantity & $\begin{array}{l}\text { At least equal to the number of operating rooms } \\
\text { o Spare lists }\end{array}$ \\
\hline Availability and accessibility & o Checklist stored at a readily accessible location in each operation room \\
\hline Checklist variants & $\begin{array}{l}\text { o Specific checklist for each surgical procedure and type of operation, task, or procedure } \\
\text { o Clear difference between variants (emergency, routine, procedure) }\end{array}$ \\
\hline \multicolumn{2}{|l|}{ Content and order } \\
\hline General & $\begin{array}{l}\text { O Tasks presented in list form in a logical, functional, or "geographical" flow } \\
\text { o Maximum of } 7 \text { tasks or checks per page recommended } \\
\text { o Long procedures separated into shorter groups if possible }\end{array}$ \\
\hline Critical items & $\begin{array}{l}\text { o Simple mnemonic used as an aid } \\
\text { o Critical items presented at the start of the tasks or clearly indicated }\end{array}$ \\
\hline \multicolumn{2}{|l|}{ Layout and format } \\
\hline Content list and index & $\begin{array}{l}\text { O Content list in the same order as the tasks } \\
\text { o Provided at the front and not exceeding } 1 \text { page, if possible } \\
\text { O An alphabetical index at the end recommended }\end{array}$ \\
\hline $\begin{array}{l}\text { Abbreviation, phraseology and } \\
\text { brevity }\end{array}$ & $\begin{array}{l}\text { O As few words as possible but understandable and unambiguous } \\
\text { o Phraseology straightforward and in standard medical terms } \\
\text { o All abbreviations standardized and explained clearly }\end{array}$ \\
\hline Start and finish & o Tasks and drills clearly defined from start to finish \\
\hline Amendments & $\begin{array}{l}\text { O Checklist construction that enables pages or cards to be changed easily for updating purpose } \\
\text { o A record of amendment state for each checklist, no longer than } 1 \text { page } \\
\text { o Amendment record page differentiated from the pages containing the tasks } \\
\text { o Each amended page dated in small print }\end{array}$ \\
\hline Physical construction & ○ Figures and tables clearly linked to the tasks \\
\hline Document size and binding & $\begin{array}{l}\text { A5 paper size with } 50 \% \text { variance } \\
\text { Binding that allows pages to be opened a minimum of } 180^{\circ} \text { and ideally up to } 360^{\circ} \text {, spiral or ring side } \\
\text { binding recommended } \\
\text { Binding such that all the text on the page can be read }\end{array}$ \\
\hline Cover & $\begin{array}{l}\text { o Cover robust and able to withstand normal handling and cleaning } \\
\text { o Cover easily distinguishable from other pages } \\
\text { o Applicable surgical procedure appearing on front of cover }\end{array}$ \\
\hline Pages and tabs/dividers & $\begin{array}{l}\text { o Pages capable of cleaning, lamination recommended } \\
\circ \text { Tabs and dividers used to assist in locating pages }\end{array}$ \\
\hline \multicolumn{2}{|l|}{ Print characteristics } \\
\hline Font type & $\begin{array}{l}\text { O Helvetica, Gill Medium, Arial, or Sans Serif recommended } \\
\text { o Font type consistent throughout the checklist } \\
\text { o Italics not used for tasks } \\
\text { o Italics acceptable for comments, notes, or supporting information }\end{array}$ \\
\hline Font size & $\begin{array}{l}\circ 14 \mathrm{pt} \text { for heading and } 12 \mathrm{pt} \text { for normal text recommended } \\
\text { O Character height-to-weight ratio of 5:3 recommended }\end{array}$ \\
\hline Margins & o Margins at least $3 / 4$ in. \\
\hline Emphasis and differentiation & $\begin{array}{l}\text { O Bold, larger font, and underlining acceptable for emphasis } \\
\text { O Small dash or bullets in front of individual tasks to aid clarity }\end{array}$ \\
\hline
\end{tabular}


Table 1 continued

\begin{tabular}{ll}
\hline Aspect & Requirement \\
\hline Other typography & $\circ$ Vertical spacing between lines not less than $25-33 \%$ of the overall size of the font \\
& $\circ$ Horizontal spacing between characters $25 \%$ of overall size and not less than one stroke width \\
& $\circ$ Good-quality printing \\
& $\circ$ Black text on white or yellow background \\
Contrast and color & $\circ$ Reflection percentage of the background should least $70 \%$ \\
& $\circ$ Luminance ratio between text and background about $1: 8$ \\
& $\circ$ Colored text and pink or red pages not recommended \\
\hline
\end{tabular}

inability to be updated automatically if items are revised or new items need to be added.

In surgical care, which focuses on the individual patient, a different strategy may be preferred. A paper-based checklist with marking can very well be used in normal circumstances, for example, as an integral part of the patient's medical chart. Critical items can be checked before a surgical procedure such as allergies, site of the operation, name of the patient, correctness of the indication, type of procedure, instruments used, and expected problems. If preferred, the checklist can be signed by the nurse or surgeon. Checking the items also can serve as a structural briefing of the entire surgical team before each procedure. Structural briefing could improve team situational awareness and enhance patient safety.

In summary, the paper-based checklist can be used for any circumstance. Because of its low technical requirements, it is easy to use and to implement in the organization. When the checklist is an integral part of the patient's medical chart, marking and signing it could solve the problem of missing items or failure to complete the checklist.

\section{Electronic or computer-based checklists}

Another type of checklist is an electronic device. A wide variety of electronic devices are available, from small digital handheld devices to a stationary desktop PC. The vocal checklist is a special variation of computer-based checklists.

The advantage of electronic checklists is that they can be updated automatically after revisions. An electronic checklist can send and receive information from compatible systems or devices. This feature enhances checklist standardization. The programmable feature and high storage capacity enable multiple checklists to be made and stored in one device. The users then can easily select the appropriate checklist for their task. Automated data capturing for research goals is another valuable feature of electronic checklists. However, initial costs and complexity are increased, and electronic devices are susceptible to system malfunction.
Another advantage of an electronic checklist is the possibility of designing an inbuilt system that prevents the start of the procedure unless the checklist is completed. This would ensure completion of the checklist.

The biggest advantage of computer-based checklists is the opportunity to feed information regarding the status of the checklist back to the user. This is called the "feedback loop." The system can alert the user if items have been missed or not completed. Rouse and Rouse [35] showed already in 1982 that pilots made significantly fewer errors using an electronic checklist than with a paper checklist [35]. Completion time, however, was longer for the electronic list, but this could be solved easily with additional training [35].

Electronic checklists that do not need a human operator also are used in aviation. Such a system was evaluated by Palmer and Degani [36]. Several levels of automation can be selected, from full manual check (users complete the checklist items) to combined check (the system completes the checklist item and subsequently asks for confirmation from the user), to fully automated check (the system completes the items without asking for user confirmation).

Several examples of electronic solutions exist. Palmtop displays are small portable electronic devices. Larger than an adult's hand, they are capable of processing and storing data and can be synchronized to a workstation. The storage capacity is smaller than that of a desktop PC.

The tablet PC is another type of portable device, and although it is larger and heavier than a palmtop display, it generally has better processing capabilities. Moreover, its larger screen provides a better display of information. Currently, a company called the Surgical Safety Institute [37] specializes in operating room safety and develops checklist software integrated in a tablet PC. However, the size of this device makes it more difficult for the users to perform other tasks with their hands while working on or holding it. If the users need a device only for a simple checklist, this device could give unnecessary trade-offs, especially for cost and portability.

Personal computers also can be used for checklists. Most modern operating rooms already have a desktop PC installed. Although its immobility can cause limitations, 
the PC can very well be used for a preoperative checklist. It requires no additional infrastructure. Moreover, with the increased use of digital patient records and operating room planning systems in hospitals, a preoperative checklist could easily be added.

The voice-controlled checklist is a sophistication of the computer-based checklist. Currently, several companies provide entire operating room concepts including voicecontrolled operation of surgical equipment, video data management, and information resources (e.g., patient records, Internet, radiology records). However, checklists are not yet included in these concepts. Furthermore, this technology is very new, and concerns about its reliability should be addressed first before it can be used safely [38].

\section{Checklist development}

Checklist development and incorporation is a systematic process. Stufflebeam [39] described a general guideline of 12 forward steps for the development of a checklist for any particular area. For the development of the surgical checklist, these steps were partially adapted and combined with our own experience, resulting in 14 steps (Fig. 1).

\section{Start checklist development}

The first step in checklist development is signaling the need for a checklist solution. In our case, a high rate of equipment problems in laparoscopic surgery during routine observations formed the motivation to develop a checklist. This step also can be used to assign a person or task force to be responsible for managing and directing the checklist development. These could be research fellows, managers, or work floor members (e.g., nurses, surgeons).

\section{Define checklist purpose}

The next step in checklist development is defining the purpose of the checklist. This step should produce a clear definition of the intended use and users of the checklist. Constructing a frequency-by-consequence table of incidents may be helpful (Fig. 2). For example, in our case, the purpose of the checklist was to minimize the equipment problem during laparoscopic surgery, and the intended users were the scrub nurses. During this step, the checklist developers can gather the necessary information by studying the relevant literature or consulting an expert of the related field.

\section{Perform a task analysis}

The third step in checklist development is performing a task analysis in the area where the checklist implementation

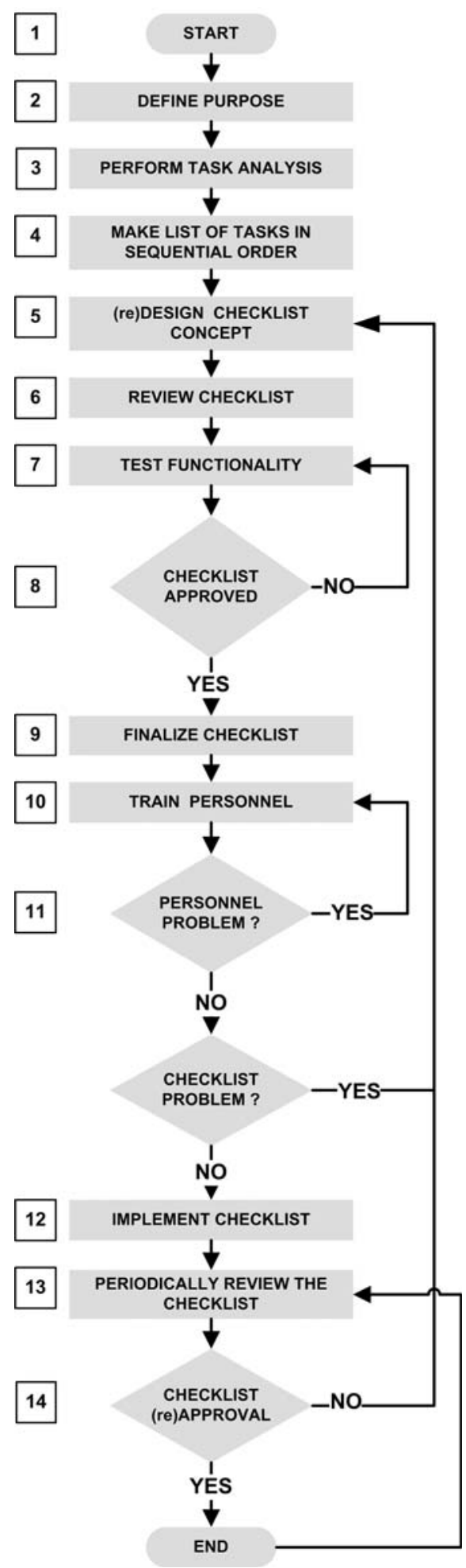

Fig. 1 Flow diagram of checklist implementation

should take place. The aim of this step is to gain an insight into the tasks of the potential users. A task analysis can be conducted in several ways such as using focus groups, direct observation, or video recordings. During focus group 


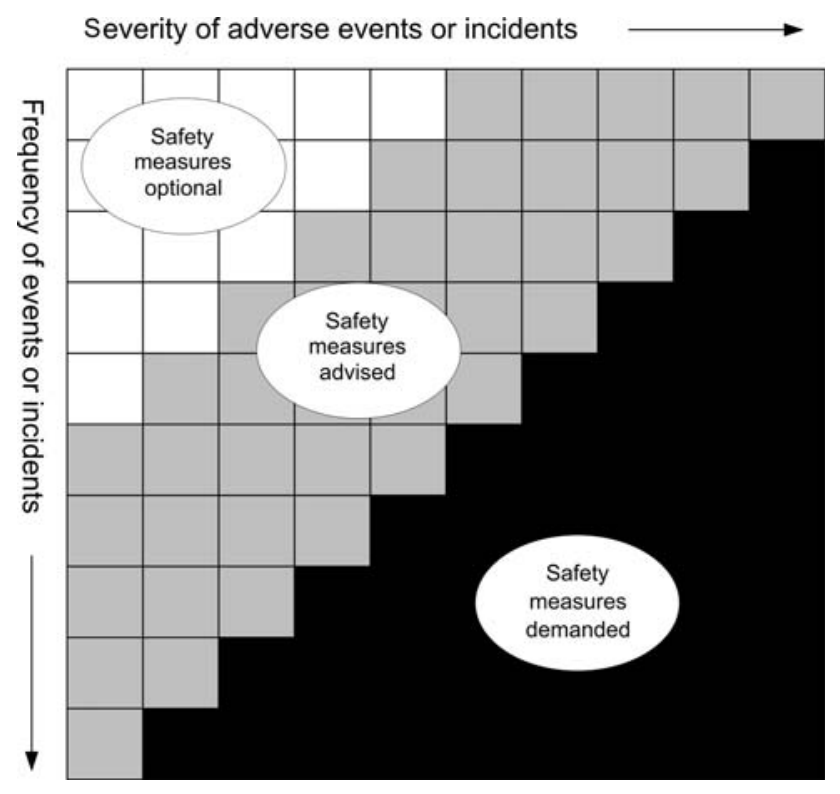

Fig. 2 Frequency-by-consequence table

sessions, all immediate stakeholders (e.g., representatives of the potential end users such as nurses, surgeons, and managers) discuss the list of activities performed. Recording the tasks in the operating room using a video camera will increase objectivity.

\section{Make a list of tasks in sequential order}

This step is closely related to the task analysis. By presenting the tasks in sequential and logical order, a clear overview of the operators' specific activities can be achieved. The task analysis report forms the basis of the checklist design.

\section{Design the checklist concept}

Next, a list of preliminary checkpoints is made based on the previous task analysis report. The end result of this step is a checklist concept. The guidelines and requirements, presented in Table 1, can be used to derive a checklist format that serves its purpose.

\section{Review the checklist (iteration)}

The checklist concept should be reviewed by all stakeholders. Ideally, the stakeholders are representatives of all the end users (the complete operating room team) and managers at the organizational level. The purpose of the review is to give feedback to the checklist developers regarding the adequacy of the checklist concept. If necessary, adjustments can be made during several iterations. The stakeholders should give approval for the checklist concept to be evaluated in the operating room or other environments.

\section{Test checklist functionality}

During a trial period, the functionality and compatibility of the checklist concept in task execution are evaluated. During this evaluation process, the checklist developers instruct several participants on how to use and complete the checklist. The participants in this case are all the intended end users. Important information regarding, for example, the attitudes of the users toward the checklist, the impact of the checklist on the existing activities, and whether the checklist serves its purpose also can be gathered during this step. Methods for collecting this information include direct or video observations and structured questionnaires.

\section{Approve checklist}

Results from the trial period are evaluated by the stakeholders. Final adjustments can be made. Then the representatives of the stakeholders formally approve the checklist concept.

\section{Prepare finalized checklist}

The checklist has now been approved and is ready to be distributed among the personnel and used in their daily work. If the checklist is paper (reusable), it is put in a more durable format to withstand frequent use. In the case of an electronic checklist, the process can be less complicated if during the development phase (steps 1 to 8), a paper checklist format is used.

\section{Train personnel}

Training personnel to use the checklist is officially initiated. All personnel are briefed about the implementation of the checklist. In the case of the preoperative safety checklist for laparoscopic equipment, the scrub nurses specifically are instructed on how to use the checklist. The surgeon is instructed to call for initiation of the checklist and confirmation of its completion. Confirming and crosschecking between different surgical team members ensures proper checklist use.

\section{Adjust checklist for personnel problems}

Although the checklist has been tested earlier, during the training sessions some new personnel-related problems can be detected. These personnel problems may lead to changes in the training method or even to checklist revisions. 
Nevertheless, if the test phase is executed properly, these will be only minor adjustments.

\section{Implement checklist}

After the minor problems have been addressed and solved, the checklist can finally be implemented. During this step, the organization and its people should be briefed on implementation of the checklist and should receive instructions.

\section{Review checklist periodically}

Organizations and tasks change constantly because new procedures or instruments are introduced. Therefore, checklists need to be reviewed periodically. The purpose of a periodic review is to evaluate the conformity of the checklist with the regulations. Basically, the frequency of checklist review is arbitrary and based on the needs of the organization, but the review should be conducted at least once a year. In addition, a checklist review should be conducted earlier if major changes in tasks, procedures, or equipment occur.

\section{(Re)approve checklist}

The result of a periodic checklist review should be used to decide whether the checklist still is acceptable until the next review. Approval is granted by one or more representatives of the stakeholders or someone on the work floor assigned to control the checklist process. If necessary, the list is revised. In the end, checklist development and implementation is a cycle that regularly evaluates the conformity of the checklist with the organization's needs and regulations.

\section{Discussion and recommendations}

Checklists as memory aids in health care are no novelty. However, literature addressing the formal use of a checklist in the operating room is scarce. Although its purpose and potential seems logical, the design of an effective checklist is not a straightforward process. In an effort to formulate design requirements for a surgical checklist, the aviation guidelines have proved to be very useful. Nevertheless, further studies are needed that translate the general guidelines proposed in this study into useful tools in daily practice. The ultimate goal is to determine the impact of checklists on the quality of care (e.g., patient safety and efficient workflow).

There is a central role for medical professionals (surgeons, nurses, and managers) in deciding how checklists should be embedded in their environment. They are the end users, and the success of the checklist approach inevitably relies on their motivation and willingness to use it. Therefore, medical professionals should be closely involved in the implementation process. Consensus is needed among medical professionals to decide which processes require a checklist, which items should be adopted, and which items should not be adopted.

It is important to underscore that adoption of the checklist approach has several important benefits especially for surgery-related healthcare that should lead ultimately to improved quality. Checklist use itself will enhance the consciousness and positive attitude toward working safely. The importance of team communication is emphasized because it will become more transparent, structured, and standardized. The tasks and responsibilities of each team member are clarified, which will enhance the objectives for team training and also for what is known as crew resource management. Crew resource management is concerned with the cognitive and interpersonal skills needed to manage the surgical procedure in a complex environment and not so much with the technical skills for actually performing the procedure.

In the past, standardization of emergency training such as for advanced trauma live support (ATLS) has proved to be successful, judging from the large number of physicians trained and the number of institutions that have adopted a similar structure [40]. Standardization of tasks, communication, and use of equipment may improve reliability of comparing interventional procedures for scientific research, and outcomes may become less dependent on the variability of individual surgical team members. However, to guarantee user commitment to a checklist, the roles and responsibilities of various team members need to be defined.

The use of checklists also could help the industry clarify equipment problems and develop effective solutions. How to monitor the effect of the checklist and provide direct feedback to the industry are not yet clear. Furthermore, research to test the benefit of checklists and to improve the (interface) design is needed.

Besides the advantages of checklist use, there are concerns for drawbacks that need to be addressed. Health care personnel may be skeptical toward the change in their work routine. Some may argue that checklist use may bring with it a significant increase in workload. Therefore, it should be made clear that the checklist approach only formalizes tasks that must be performed anyway. Additional work to complete checklists should be kept at a minimum, so each checklist should be carefully designed. Furthermore, checklists causing superfluous administration should be avoided. This is, however, no argument against a paper checklist. In addition, it is most likely that future checklists 
will be electronic because the evolution in all hospitals goes in the direction of a no paper patient file.

Measurements to increase safety require financial investments and can be costly at first. However, in the long run, these costs will be compensated largely by improvements in quality of care. Nevertheless, it is not expected that all investments in the checklist can be translated directly into measurable improvements. The effect of checklists is highly dependent on influences of the "safety culture" within a certain environment. Furthermore, as Calland et al. [5] have already suggested, checklists are only one of many strategies to improve quality and institute a safety culture. Strategies such as systematic monitoring of incidents, in-depth accident investigation (root cause analysis), and structural and continued training based on objective assessment are important as well. In addition, checklists are not watertight in preventing human error or accidents in general. Their strength lies in the combination of various safety measurements, as mentioned earlier.

In conclusion, the use of structured checklists in surgical processes is most likely to be effective because it standardizes human performance and ensures that procedures are followed correctly instead of relying on human memory alone. Several studies present promising and positive first results, providing a solid basis for further investigation. Future research should focus on the effect of various checklist designs and strategies to ensure maximal compliance.

Acknowledgment This research was supported financially by a grant provided by the health insurance company DSW (Schiedam, The Netherlands).

Open Access This article is distributed under the terms of the Creative Commons Attribution Noncommercial License which permits any noncommercial use, distribution, and reproduction in any medium, provided the original author(s) and source are credited.

\section{References}

1. Kohn LT, Corrigan JM, Donaldsen MS (1999) To err is human. Institute of Medicine, Washington DC, pp 1-14

2. Wagner C, de Bruijne M (2007) Onbedoelde schade in Nederlandse ziekenhuizen. Nederlands Instituut voor onderzoek van de gezondheidszorg (NIVEL) p 20

3. Leape L (1994) The preventibility of medical injury. In: Bogner MS (ed) Human error in medicine. Lawrence Erlbaum Associates, Hillsdale, $\mathrm{NJ}$

4. Reason J (2000) Human error: models and management. BMJ 320:768-770

5. Calland JF, Guerlain S, Adams RB, Tribble CG, Foley E, Chekan EG (2002) A systems approach to surgical safety. Surg Endosc 16:1005-1014 discussion 1015

6. Dankelman J, Grimbergen CA (2005) Systems approach to reduce errors in surgery. Surg Endosc 19:1017-1021
7. Vincent C, Moorthy K, Sarker SK, Chang A, Darzi AW (2004) Systems approaches to surgical quality and safety: from concept to measurement. Ann Surg 239:475-482

8. Helmreich RL (2000) On error management: lessons from aviation. BMJ 320:781-785

9. Undre S, Healey AN, Darzi A, Vincent CA (2006) Observational assessment of surgical teamwork: a feasibility study. World J Surg 30:1774-1783

10. Verdaasdonk EG, Stassen LP, van der Elst M, Karsten TM, Dankelman J (2007) Problems with technical equipment during laparoscopic surgery: an observational study. Surg Endosc $21: 275-279$

11. Saufl NM (2004) Universal protocol for preventing wrong-site, wrong-procedure, wrong-person surgery. J Perianesth Nurs $19: 348-351$

12. Universal protocol for preventing wrong-site, wrong-procedure, wrong-person surgery. Retrieved June 2007 at www.joint commission.org/PatientSafety/UniversalProtocol/]

13. Kwaan MR, Studdert DM, Zinner MJ, Gawande AA (2006) Incidence, patterns, and prevention of wrong-site surgery. Arch Surg 141:353-357 discussion 357-358

14. Michaels RK, Makary MA, Dahab Y, Frassica FJ, Heitmiller E, Rowen LC, Crotreau R, Brem H, Pronovost PJ (2007) Achieving the National Quality Forum's "Never Events": prevention of wrong-site, wrong-procedure, and wrong-patient operations. Ann Surg 245:526-532

15. Lingard L, Espin S, Rubin B, Whyte S, Colmenares M, Baker GR, Doran D, Grober E, Orser B, Bohnen J, Reznick R (2005) Getting teams to talk: development and pilot implementation of a checklist to promote interprofessional communication in the OR. Qual Saf Health Care 14(5):340-346

16. Makary MA, Mukherjee A, Sexton JB, Syin D, Goodrich E, Hartmann E, Rowen L, Behrens DC, Marohn M, Pronovost PJ (2007) Operating room briefings and wrong-site surgery. J Am Coll Surg 204:236-243

17. Makary MA, Holzmueller CG, Thompson D, Rowen L, Heitmiller ES, Maley WR, Black JH, Stegner K, Freischlag JA, Ulatowski JA, Pronovost PJ (2006) Operating room briefings: working on the same page. Jt Comm J Qual Patient Saf 32(6):351-355

18. Leonard M, Graham S, Bonacum D (2004) The human factor: the critical importance of effective teamwork and communication in providing safe care. Qual Saf Health Care 13(Suppl 1):i85-i90

19. DeFontes J, Surbida S (2004) Preoperative safety briefing project. Permanente J 8:21-27

20. Berge JA, Gramstad L, Grimnes S (1994) An evaluation of a time-saving anaesthetic machine checkout procedure. Eur $\mathrm{J}$ Anaesthesiol 11:493-498

21. Barthram C, McClymont W (1992) The use of a checklist for anaesthetic machines. Anaesthesia 47:1066-1069

22. March MG, Crowley JJ (1991) An evaluation of anesthesiologists' present checkout methods and the validity of the FDA checklist. Anesthesiology 75:724-729

23. Manley R, Cuddeford JD (1996) An assessment of the effectiveness of the revised FDA checklist. Aana J 64:277-282

24. Berge JA, Gramstad L, Jensen O (1993) A training simulator for detecting equipment failure in the anaesthetic machine. Eur $\mathrm{J}$ Anaesthesiol 10:19-24

25. Blike G, Biddle C (2000) Preanesthesia detection of equipment faults by anesthesia providers at an academic hospital: comparison of standard practice and a new electronic checklist. Aana J 68:497-505

26. Kendell J, Barthram C (1998) Revised checklist for anaesthetic machines. Anaesthesia 53:887-890

27. Hart EM, Owen H (2005) Errors and omissions in anesthesia: a pilot study using a pilot's checklist. Anesth Analg 101:246-250. table of contents 
28. Meijer DW (2003) Safety of the laparoscopy setup. Minim Invasive Ther Allied Technol 12:125-128

29. Cival Aviation Authority (CAA) (2000) Guidance on the design, presentation, and use of electronic checklists. CAP 708. Safety Regulation Group. Retrieved July 2008 at http://www.caa.co.uk/ docs/33/CAP708.PDF

30. Cival Aviation Authority (CAA) (2006) Guidance on the design presentation and use of emergency and abnormal checklists. CAP 676. Safety Regulation Group. Retrieved July 2008 at http://www.caa.co.uk/docs/33/CAP676.PDF

31. Degani A, Wiener EL (1990) Human factors of flight deck checklists: the normal checklist. NASA Contractor Report 177549

32. Reason J (1990) Human error. Cambridge University Press

33. Degani A, Wiener EL (1993) Cockpit checklists: concepts, design, and use. Hum Factors 35(2):28-43

34. Diamond T, Mole DJ (2005) Anatomical orientation and crosschecking, the key to safer laparoscopic cholecystectomy. Br J Surg 92:663-664
35. Rouse SH, Rouse WB (1980) Computer-based manuals for procedural information. IEEE Trans Syst Man Cybern 10(8):506510

36. Palmer E, Degani A (1994) Electronic checklists: evaluation of two levels of automation. In: Proceedings of the Sixth International Aviation Psychology Symposium. Ohio State Univeristy, Columbus, $\mathrm{OH}$

37. www.surgicalsafetyinstitute.com

38. Punt MM, Stefels CN, Grimbergen CA, Dankelman J (2005) Evaluation of voice control, touch panel control, and assistant control during steering of an endoscope. Minim Invasive Ther Allied Technol 14:181-187

39. Stufflebeam DL (2000) Guidelines for developing evaluation checklists: the checklists development checklist (CDC). Retrieved May 2007 at http://www.wmich.edu/evalctr/checklists/ guidelines_cdc.pdf]

40. Gwinnutt CL, Driscoll PA (1996) Advanced trauma life support. Eur J Anaesthesiol 13:95-101 